\title{
2. Spotlight: Monitoring, die kritische Beobachtung einer in Teilen umstrittenen Technologie
}

Vor ca. zwanzig Jahren etablierte die Berlin-Brandenburgische Akademie der Wissenschaften ein Monitoringsystem zur Beobachtung der Gentechnologie in Deutschland. Eine Gruppe von Wissenschaftlerinnen und Wissenschaftlern verschiedener Fachrichtungen, eine sog. Interdisziplinäre Arbeitsgruppe (IAG), sollte Entwicklungen und Verlauf einer damals noch jungen und umstrittenen Hochtechnologie analysieren und kritisch begleiten. Nach der Publikation von vier Gentechnologieberichten und mehreren Themenbänden soll hier der Kernbereich des Monitoringsystems der BBAW im Rückblick exemplarisch betrachtet werden.

Ursprünglich kommt der Begriff aus dem Bereich der Technik: Ein Monitor ist ein Gerät, das als Teil eines technischen Prozesses bestimmte Parameter registriert, überwacht und reguliert. Der Verlauf und potenzielle Erfolg des Prozesses werden somit abgebildet und gesteuert. Inzwischen wurde seine Anwendung wesentlich erweitert und bezieht sich heute u. a. auf biologische, ökologische, wirtschaftliche (z. B. Marketing-) und politische Vorgänge in Natur und Gesellschaft. Während das Monitoring eines Prozesses - etwa einer Produktion, einer Entwicklung, eines Verfahrens - Grundlage der Steuerung jener Vorgänge war, kann die Aufgabe einer IAG, wie hier beschrieben, eher die einer passiven Beobachtung, eines Observatoriums mit beratender Funktion sein, wie sie kürzlich von Jasanoff und Hurlbut für das Gene Editing vorgeschlagen wurde (Jasanoff/Hurlbut, 2018). Insbesondere durch die Bereitstellung von Kernaussagen und Handlungsempfehlungen geht die IAG allerdings über eine reine ergebnisoffene Beschreibung der Sachlage hinaus und wird von der Öffentlichkeit insofern nicht nur als Beobachterin, sondern auch als beratendes Gremium wahrgenommen (Könninger, 2018). 


\subsection{Monitoring der Gentechnologie in Deutschland}

Der erste Gentechnologiebericht der BBAW erschien 2005 (Hucho et al.). Das Monitoring der IAG wurde zunächst auf vier Themenbereiche beschränkt, um die Komplexität der Aufgabe zu begrenzen; auf die Anwendung der Gentechnologie in der Grundlagenforschung (Fallbeispiel Genomforschung), auf die Anwendung in der Medizin (Fallbeispiel molekulargenetische Diagnostik), auf die Anwendung in der Agrarwirtschaft (Fallbeispiel Pflanzenforschung) und schließlich auf die ökonomische Bedeutung (Fallbeispiel Biotech-Start-ups). Leitbegriffe sollten eine Reihe von Querschnittsdimensionen sein, wie z. B. ethische, juristische, ökologische, ökonomische und gesellschaftliche Aspekte. Es schwebte uns somit eine Matrix aus speziellen Themen und prinzipiellen Betrachtungsweisen vor. Dieses Schema erwies sich als zu starr, zumal je nach Thema die Datenlage verhinderte, ein Standardschema durchzuhalten. Auf daraus resultierende Schwierigkeiten wird weiter unten eingegangen.

Oberster Gesichtspunkt war jedoch durchgehend die Interdisziplinarität der Analyse. Sie wurde durch die Fachkunde der Gründungsmitglieder des Projektes sichergestellt. Ohne die einzelnen Kolleginnen und Kollegen auf ein enges Gebiet festzulegen, seien hier nur die Namen genannt. Mitglieder, Herausgeberinnen und Herausgeber des ersten Gentechnologieberichts waren Klaus Brockhoff, Wolfgang van den Daele, Kristian Köchy, Jens Reich, Hans-Jörg Rheinberger, Bernd Müller-Röber, Karl Sperling, Anna M. Wobus, Mathias Boysen, Maike Kölsch und als Initiator und Sprecher der Gruppe Ferdinand Hucho, also eine intellektuelle Vielfalt aus Molekularbiologen, Philosophen, Soziologinnen und Soziologen, einer Zellbiologin, einem Ökonomen, einem Pflanzenphysiologen und einem Biomediziner. Es fehlte zunächst eine Juristin oder ein Jurist, ein Manko, das später durch die Zuwahl von Jochen Taupitz beseitigt wurde.

\subsection{Werkzeug des Monitorings: Indikatoren}

Wichtigste Komponente eines Monitoringsystems ist der Monitor, das eigentliche Messinstrument.

Alleinstellungsmerkmal des Gentechnologieberichts ist das Monitoring vermittels Indikatoren. Idealerweise ist ein Indikator eine Messgröße, eine quantifizier- und vergleichbare Eigenschaft des Gegenstandes des Monitorings.

Ein triviales Beispiel: Die Bedeutung der Gentechnologie für die Grundlagenforschung lässt sich als solche nicht messen. Bricht man sie jedoch in Teilgrößen auf wie z. B. die Anzahl der Publikationen mit gentechnischem Hintergrund, die Anzahl der auf diesem Gebiet aktiven Wissenschaftlerinnen und Wissenschaftler, der öffentlichen Fördermittel, der Patente usw., so erhält man Zahlenwerte, die es ermöglichen, den 
abstrakten Begriff Bedeutung zu konkretisieren. Vor allem lassen sich Zeitverläufe und Entwicklungen in Graphen darstellen.

Der wohl bekannteste Indikator auf ökonomischem Gebiet ist das Bruttosozialprodukt. Er beschreibt die Wirtschaftsleistung einer Volkswirtschaft, eines Staates. Tatsächlich ist er ein zusammengesetzter Indikator, der die Summe der Dienst-, Arbeitsund Produktionsleistungen in einem Jahr angibt. Für die Gentechnologie gelang es bisher nicht, einen solcherart zusammengesetzten Indikator zu formulieren. Es wäre eine lohnende Aufgabe für qualifizierte Soziologinnen und Soziologen, hier kreativ zu werden.

Die Qualität eines Indikators hängt von der Datenbasis ab: Der Gentechnologiebericht basiert nicht auf von der IAG selbst erhobenen Daten, sondern verwendet ausschließlich Datenbanken des Internets. Deren Qualität kann sehr unterschiedlich sein. $\mathrm{Zu}$ Anfang der Arbeit der IAG wurde daher versucht, eine „Metadatenbank“ zu schaffen, eine Sammlung von bewerteten Banken. Dies erwies sich jedoch als so aufwendig, dass es aufgegeben werden musste.

\subsection{Problemfeldanalyse ${ }^{1}$}

Um die Aussagekraft des „Alleinstellungsmerkmals Indikator“ zu steigern, entwickelten M. Boysen und M. Kölsch, wissenschaftliche Mitarbeitende der BBAW-Geschäftsstelle des Monitoringprojektes „Gentechnologiebericht“, ein graphisches Verfahren, das dazu dient, die Gewichtung der Indikatoren im Umfeld öffentlicher Aufmerksamkeit sichtbar zu machen. Die Problemfeldanalyse erwies sich als nützlich, sodass sie in die Systematik des Gentechnologieberichtsprojektes aufgenommen, weiterentwickelt und ausdifferenziert ${ }^{1}$ wurde (Boysen/Kölsch, 2006; Marx-Stölting/Könninger, 2018) und sich für Monitoringprojekte im Allgemeinen empfiehlt. Hierzu werden z. B. vier relevante Dimensionen definiert, die ein Achsenkreuz ergeben. Sie werden aus der öffentlichen Diskussion in maßgeblichen Medien abgeleitet. Für die Gentechnologie könnten diese Dimensionen sein: 1 . die wissenschaftliche, 2. die ethische, 3. die soziale und 4. die ökonomische Dimension des Themas. In dieses Achsenkreuz trägt man nun Problemfelder ein, die sich durch Indikatoren belegen lassen und ordnet sie je nach ihrem Bezug in Nachbarschaft der jeweiligen Eckpunkte des Achsenkreuzes ein. Aus der Verteilung der Problemfelder über die Fläche, die durch das Achsenkreuz vorgegeben wird, lässt sich rein optisch die Relevanz bestimmter Probleme für das gesellschaftliche Um-

1 Für einen Überblick siehe Marx-Stölting/Könninger (2018) sowie das Indikatorenkapitel von Osterheider et al. in diesem Bericht. 
feld ableiten. Selbst dann, wenn einzelne Problemfelder nicht durch Indikatoren exakt quantitativ belegbar sind, enthält diese optische Darstellung wertvolle Informationen. Seit 2011 wird die Häufigkeit der Erwähnung der Problemfelder in den ausgewerteten Quellen zusätzlich über Größe und/oder Färbung der Problemfeldblasen in dieser Übersichtsgrafik dargestellt (für eine ausführliche Darstellung der Problemfeldanalyse siehe Boysen/Kölsch, 2006).

\subsection{Allgemeine Voraussetzungen für das Monitoring}

Wichtig ist es, die Zielgruppe des Monitorings zu definieren: Handelt es sich um die „breite Öffentlichkeit“, muss wissenschaftlicher Fachjargon vermieden werden. Soll ein Datenkorpus für Fachwissenschaftlerinnen/Fachwissenschaftler generiert werden, kommt es eher auf die Präzision der Fachsprache an.

Ebenso wichtig ist die Unvoreingenommenheit der Autorinnen und Autoren. Sie sollten kein Eigeninteresse am Gegenstand der Analyse haben, das über das einer Fachwissenschaftlerin/eines Fachwissenschaftlers hinausgeht. Die Autorinnen und Autoren sollten als Gruppe interdisziplinär aufgestellt sein. Es sollten Daten und Fakten für einen ergebnisoffenen Diskurs zur Verfügung gestellt werden.

\subsection{Umfeld des Monitorings: die interdisziplinäre Arbeitsgruppe und ihre Arbeitsweise}

Es war zunächst vorgesehen, in einem festen Zwei- bis Vierjahresrhythmus einen Gentechnologiebericht vorzulegen, der auf der Basis von Indikatoren Entwicklungen sichtbar macht und fortschreibt. Dies erwies sich wegen der Dynamik des wissenschaftlichen Fortschritts jedoch als nicht durchführbar. Um neue Entwicklungen aktuell abzuhandeln, wurden zeitlich zwischen den Berichten Themenbände veröffentlicht, die spezielle Gegenstände der Gentechnologie darstellten: Stammzellforschung, Molekulare Diagnostik, Gentherapie, synthetische Biologie, Grüne Gentechnologie, Epigenetik, Organoide - um nur die wichtigsten zu nennen. ${ }^{2}$ Außerdem wurden zwei Analysen zu den Themen Genomchirurgie und Einzelzellanalysen sowie zwei Sonderausgaben der Zeitschrift Journal of Molecular Medicine zu Stammzellen und Organoiden herausgebracht. Neben dem naturwissenschaftlichen Sachstand und den Indikatoren wurden in die Publikationen stets auch rechtliche, ethische, wissenschaftstheoretische und histo-

2 Für eine vollständige Publikationsliste siehe: www.gentechnologiebericht.de/publikationen [05.02.2021]. 
rische Beiträge aufgenommen, um solche Bereiche abzudecken, die mittels Indikatoren nicht quantifizierbar waren und einer zusätzlichen qualitativen Beschreibung bedurften. Dies spiegelt sich auch im vorliegenden „Fünften Gentechnologiebericht“ wider.

Über die reine Publikationstätigkeit hinaus organisierte die IAG zahlreiche Veranstaltungen und Vortragsreihen zu ihren Themenbereichen, um die Diskussion in der Öffentlichkeit anzuregen.

\subsection{Erfahrungen mit der Systematik des Monitorings und essenzielle Grundlagen}

Die umfangreiche Publikationsliste der IAG verdeutlicht, dass sich das hier skizzierte Monitoringsystem, so wie es über die Jahre hin ausgearbeitet und optimiert wurde, bewährt hat.

Wichtig für das Monitoring generell sind:

- die Fachkunde und Interdisziplinarität der Arbeitsgruppe. Beides ist optimal im Rahmen einer wissenschaftlichen Akademie vorhanden, wobei je nach Thema und Situation externe Expertinnen und Experten die Gruppe der Akademiemitglieder ergänzen können.

- Wichtig ist ferner ein Monitoringsystem, beruhend auf Indikatoren, die je nach Datenlage (Internet) ausgewählt und in eine Problemfeldanalyse eingebracht werden (aber siehe unten).

- Wichtig ist eine klare Gliederung der Arbeit: Der wissenschaftliche Sachstand des Gegenstands des Monitorings muss ausführlich, präzise und ohne Voreingenommenheit dargestellt werden.

- Die Indikatoren unterstreichen eher die wissenschaftliche Fundiertheit der Analysen. Für die Adressatin oder den Adressaten und die Nutzerin oder den Nutzer der Analyse ist meistens wichtiger, dass Handlungsempfehlungen - sei es in Bezug auf notwendige Fördermaßnahmen, sei es in Hinblick auf zu erforschende wissenschaftliche Grundlagen - gegeben werden.

- Ein längerfristiges Monitoring bedarf der flexiblen Anpassung, sowohl der Auswahl und Abgrenzung des Gegenstands des Monitorings, der Systematik und Herangehensweise, der Indikatoren, als auch der Auswahl der Expertinnen und Experten, der Autorinnen und Autoren. 


\subsection{Literaturverzeichnis}

Boysen, M./Kölsch, M. (2006): Methodischer Ansatz für eine systematische Betrachtung der Stammzellforschung. In: Wink, R. (Hrsg.): Deutsche Stammzellpolitik im Zeitalter der Transnationalisierung. Nomos, Baden-Baden: 87-103.

Domasch, S./Boysen, M. (2007): Problemfelder im Spannungsfeld der Gendiagnostik. In: Schmidtke, J. et al. (Hrsg.): Gendiagnostik in Deutschland. Status quo und Problemerkundung. Forum W, Limburg: 179-187.

Hucho, F. et al. (2005): Gentechnologiebericht. Analyse einer Hochtechnologie in Deutschland. Elsevier Spektrum, München.

Jasanoff, S./Hurlbut, J. B. (2018): A global observatory for gene editing. Nature 555: 435-437.

Könninger, S. (2018): Ein Monitoring monitoren - die IAG Gentechnologiebericht in der Wahrnehmung der medialen Öffentlichkeit. In: Hucho, F. et al. (Hrsg.): Vierter Gentechnologiebericht. Nomos, Baden-Baden: 349-375.

Marx-Stölting, L./Könninger, S. (2018): Problemfelder gestern und heute. In: Hucho, F. et al. (Hrsg.): Vierter Gentechnologiebericht. Nomos, Baden-Baden: 279-297. 